\title{
PROGRAMA DE CÁLCULO NUMÉRICO EM VISUAL BASIC FOR APPLICATIONS
}

DOI: 10.37702/2175-957X.COBENGE.2021.3512

João Victor Cancela Sousa - jvcancela19@gmail.com

Universidade Federal do Pará

Rodovia Mário Covas 1835

67113-330 - Ananindeua - PA

Arthur Felipe dos Santos Fernandes - arthurengl.ufpa@gmail.com Universidade Federal do Pará

Vila Rosa 66

66093-470 - Belém - PA

LUCAS MANOEL MORAES PORTAL - lucasmano230@gmail.com Universidade Federal do Pará

Travessa: D 20

67115-790 - Ananindeua - PA

valmir passos da silva - valmir100passos1@gmail.com

Universidade Federal do Pará

Orlando Fonseca Silva - orfosi@ufpa.br

Universidade Federal do Pará

Passagem Alberto Engelhard 124

66040-520 - Belém - PA

Resumo: Cálculo Numérico é o estudo de métodos que servem para se obter a solução de problemas matemáticos de forma aproximada. Invariavelmente envolvem um grande número de cálculos aritméticos inviabilizando a realização manual em grande escala. Contudo com o desenvolvimento de computadores digitais eficientes, tornou-se possível realizar esses cálculos mais extensos de forma rápida e eficiente. Objetivando contribuir de forma prática e dinâmica no processo ensino aprendizagem da disciplina Cálculo Numérico, desenvolveu-se um aplicativo em Visual Basic for Applications (VBA) para a solução de vários 


\section{COBENCE - Evento onine

problemas típicos apresentados na disciplina.

Palavras-chave: Algoritmo; Aplicativo; Programação 


\section{PROGRAMA DE CÁLCULO NUMÉRICO EM VISUAL BASIC FOR APPLICATIONS}

\section{INTRODUÇÃO}

A disciplina Cálculo Numérico agrega conteúdos importantes ao conhecimento do aluno, pois apresenta métodos para se obter soluções de problemas matemáticos de forma aproximada a partir de um determinado número de iterações. O caráter iterativo torna essas soluções adequadas para implementação em computadores digitais.

Nesse sentido, desenvolveu-se um aplicativo, com o intuito de contribuir no ensino aprendizagem e trazer praticidade aos alunos no estudo da disciplina em questão, pois ao utilizá-lo, é possível avaliar se a resposta de seus exercícios está correta ou verificar rapidamente o impacto na solução quando da alteração de parâmetros envolvidos.

\section{DESENVOLVIMENTO DO PROGRAMA}

Aplicativos computacionais são desenvolvidos em uma linguagem de programação para realizar um conjunto de operações especificadas pelo programador. Cada linguagem possui suas especificidades e dependendo da situação uma delas acaba se tornando mais viável que as outras.

\subsection{Linguagem de programação utilizada}

Para o desenvolvimento do aplicativo escolheu-se como linguagem de programação o Visual Basic For Applications (VBA), que faz parte do pacote Microsoft Office e está disponível na aba de desenvolvedor do Excel (deve estar instalado no computador) que é o editor de planilhas da empresa.

A escolha deve-se aos seguintes fatores: maior disponibilidade deste em computadores quando comparados aos softwares específicos para exercer tarefas relacionadas a cálculo matemático; e também em função da possibilidade de desenvolver uma interface mais agradável, permitindo a criação de janelas e a inserção de botões para a seleção da ferramenta pretendida, além de realizar o cálculo após inserir os dados nos espaços apropriados.

\subsection{Metodologia para o desenvolvimento do programa}

Durante as aulas da referida disciplina, foram realizadas anotações dos métodos e dos exercícios resolvidos. Nos métodos observou-se que cada um deles podia ser representado por um modelo matemático, sendo possível fazer a programação dos mesmos.

Para o desenvolvimento do aplicativo objetivou-se inserir janelas e botões visando tornar a interface mais intuitiva ao estudante da disciplina.

A partir dos exemplos feitos em sala de aula, fez-se a programação passo a passo de cada método durante o período letivo das aulas da disciplina. Para tanto, estruturas de repetição e condicionais foram utilizadas, fosse pela característica do método ou para diminuir a quantidade de linhas de código no programa tornando assim o aplicativo mais otimizado. 


\subsection{Ferramentas do aplicativo}

O aplicativo possui as seguintes ferramentas: determinação de raízes de polinômios, resolução de sistemas lineares, determinação do polinômio interpolador, derivação e integração numérica.

\section{Determinação das raízes de polinômios}

As raízes de um polinômio são denotadas como os valores da variável independente que fazem com que o polinômio seja igual a zero. Entre os métodos presentes no aplicativo têm-se: o método da bisseção, posição falsa e secante.

\section{Resolução de sistema linear}

Um conjunto finito de equações lineares, aplicadas a um conjunto finito de variáveis formam um sistema linear. $O$ aplicativo resolve sistemas lineares que tenham solução possível e única. Entre os métodos presentes têm-se: Gauss-Jordan, inversa de matriz, fatoração LU, Cramer, Gauss-Jacobi e o Gauss-Seidel.

\section{Determinação do polinômio interpolador}

Dado um conjunto de pontos em um sistema de eixos cartesianos, o processo de determinação de um polinômio que passe por todos os pontos é de denominado interpolação polinomial. Entre os métodos presentes no aplicativo têm-se: Newton, Lagrange e o método da solução de sistema.

\section{Derivação numérica}

A diferenciação numérica descreve algoritmos para calcular a derivada de uma função matemática. Entre os métodos presentes no aplicativo estão a derivação numérica regressiva, central e progressiva.

\section{Integração numérica}

A integração numérica tem o objetivo de aproximar o resultado de integrais definidas. Entre os métodos presentes no aplicativo têm-se: trapézio, trapézio repetido, Simpson e Simpson repetido.

\subsection{Limitações}

Apesar das vantagens que o VBA apresenta como a maior disponibilidade em computadores, a possibilidade de criar abas com a interface didática para os estudantes da disciplina, é necessário pontuar as dificuldades e limitações no desenvolvimento.

A determinação de raízes limita-se apenas a funções polinomiais até o quarto grau, quando na prática deveria abranger outras funções como, por exemplo, as trigonométricas e não ter restrição quanto ao grau. Tais restrições foram devidas a complexidade da implementação na plataforma.

Outro exemplo está nas ferramentas de resolução de sistemas lineares onde estes podem ter no máximo 4 variáveis, pois o aumento na quantidade de incógnitas torna os cálculos mais extensos inviabilizando essa opção.

\subsection{Funcionamento}

O ícone da "Figura 1" ilustra o arquivo na área de trabalho. Uma vez aberto o aplicativo pela primeira vez o usuário deverá clicar em "habilitar conteúdo" em uma mensagem que aparecerá em destaque na aba do Excel como mostra a "Figura 2".

Figura 1 - Aparência do arquivo na área de trabalho. 
Programa de calculo Numerico

Fonte: Os autores

Figura 2 - Aviso para habilitar o conteúdo.

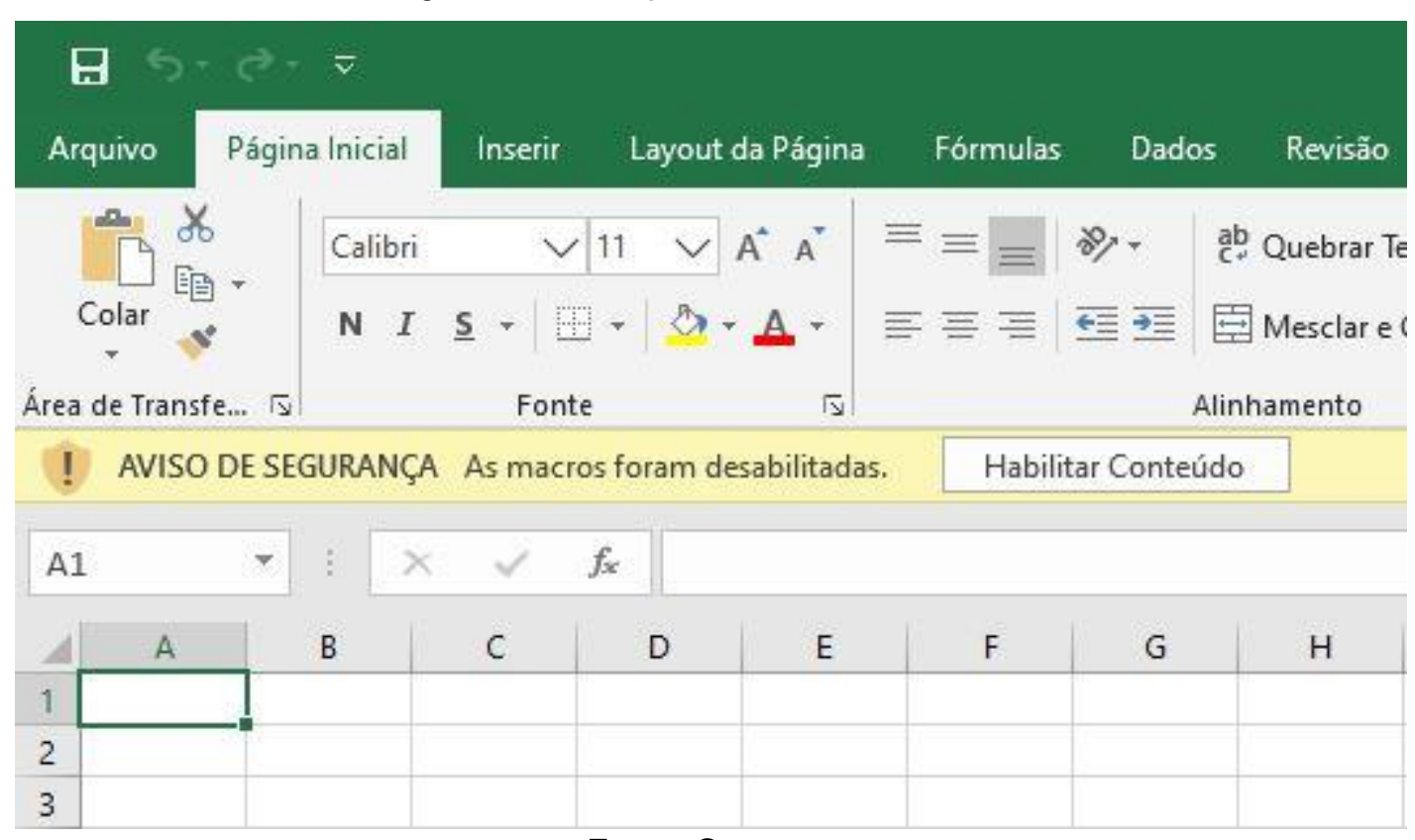

Fonte: Os autores

Em seguida o usuário poderá selecionar a ferramenta e o método que deseja realizar a partir da interface ilustrada na "Figura 3".

Figura 3 - Ferramenta desenvolvida 
Selecione a ferramenta que deseja utilizar:

\begin{tabular}{|c|}
\hline Encontrar raízes pelo método da bisseção \\
\hline Encontar raízes pelo método da posição falsa \\
\hline Encontar raízes pelo método da secante \\
\hline Resolução de sistema linear pelo método de Gauss-Jordan \\
\hline Resolução de sistema linear pelo método da inversa da matriz \\
\hline Resolução de sistema linear pelo método da fatoracao LU \\
\hline Resolução de sistema linear pelo método de Cramer \\
\hline Resolução de sistema linear pelo método de Gauss-Jacobi \\
\hline
\end{tabular}

Polinômio interpolador pelo método da solução de sistema

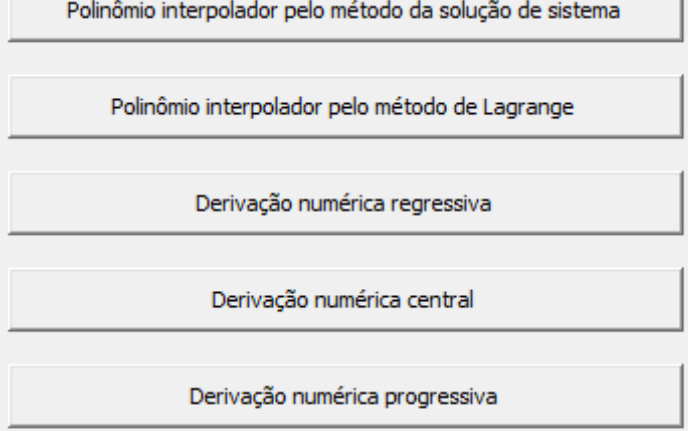

Integração numérica pelo método do Trapézio

Integração numérica pelo método do Trapézio repetido

Integração numérica pelo método de Simpson

Fonte: Os autores

Por exemplo, ao escolher a ferramenta de resolução de sistema linear pelo método de Cramer, uma nova janela, conforme a "Figura 4", permitirá a seleção do número de incógnitas.

Figura 4 - Seleção do número de incógnitas do sistema linear

Resolução de sistemas lineares pelo método de Cramer

$\times$

Selecione o número de incógnitas do sistema linear:

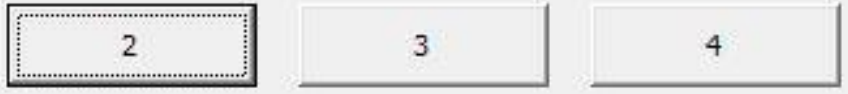

Fonte: Os autores

Após selecionar o número de variáveis, uma nova aba é apresentada. Nela serão inseridos os coeficientes do sistema linear com variáveis arbitrariamente nomeadas de " $x 1$ " e "x2". Como exemplo a primeira linha do sistema está descrita na "Equação (1)" e a segunda na "Equação (2)". 
Equação 1:

$$
7 x_{1}-2 x_{2}=3
$$

Equação 2:

$$
3 x_{1}+1 x_{2}=5
$$

Ao selecionar "Calcular" a resposta é exibida ao lado, onde os resultados para os valores de "x1" e "x2" desse exemplo são respectivamente 1 e 2 de acordo com a "Figura $5 "$.

Figura 5 - Janela para inserção dos valores.

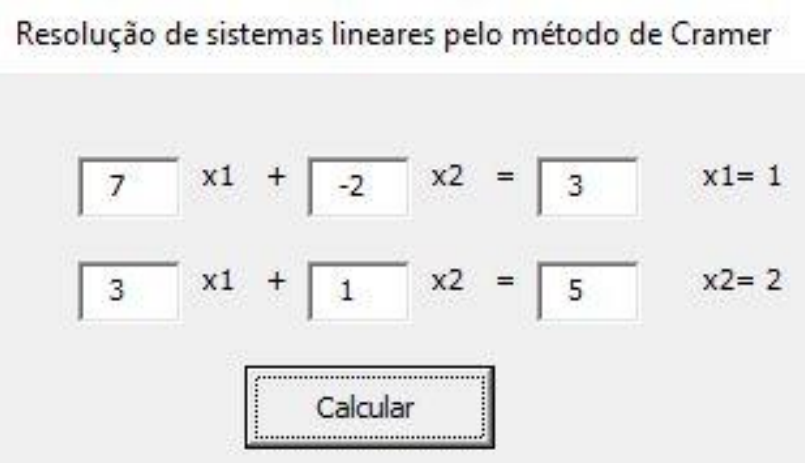

Fonte: Os autores

É válido ressaltar que a construção deixa o programa mais intuitivo e de fácil visualização por parte do aluno. Cada método solicita ao usuário as informações do exercício de acordo com as especificidades de cada um, por exemplo alguns solicitam o número de pontos para a interpolação polinomial, outros solicitam o grau da função polinomial para encontrar as raízes, entre outros. Os outros métodos seguem a mesma estrutura no que concerne as janelas para seleção das configurações do método.

\subsection{Avaliação do programa por alunos da disciplina}

Em função da pandemia de COVID-19 a avaliação foi feita de forma online por meio de questionário no Google Forms. No início do período letivo os estudantes da disciplina foram convidados a participar da pesquisa por e-mail, e os que manifestaram interesse receberam o aplicativo para teste e puderam entender o funcionamento dos métodos do programa conforme a disciplina era desenvolvida pelo(a) professor(a) ao longo do período letivo. Ao final da disciplina os estudantes receberam o formulário, que foi respondido de forma anônima. O questionário era composto de duas perguntas de múltipla escolha, além de um terceiro item para o estudante deixar caso quisesse um feedback de forma escrita.

A turma do semestre analisado envolvia 30 estudantes, somente 6 demostraram interesse e responderam o e-mail convidando para a participação da pesquisa durante 0 semestre, desses apenas 3 responderam ao questionário enviado após a conclusão da disciplina, possivelmente a participação do público seria maior em um período de aulas presenciais na universidade. 
A primeira pergunta estava relacionada ao entendimento do funcionamento do programa durante o período, e $66,7 \%$ dos participantes responderam que conforme 0 conteúdo da disciplina avançava era possível compreender melhor o que representava cada parte de entrada e saída de dados e os outros $33,3 \%$ responderam que conseguiram entender como utilizar o aplicativo, porém em alguns métodos não obtiveram êxito nessa tarefa, como, por exemplo, na "Figura 6" que ilustra o método da bisseção que é voltado para encontrar as raízes de polinômios de grau maior que 2, onde deve-se inserir um intervalo e o número de iterações, o que fica difícil compreender do que se trata antes de ter uma aula ou estudar a respeito.

Figura 6 - Método da bisseção

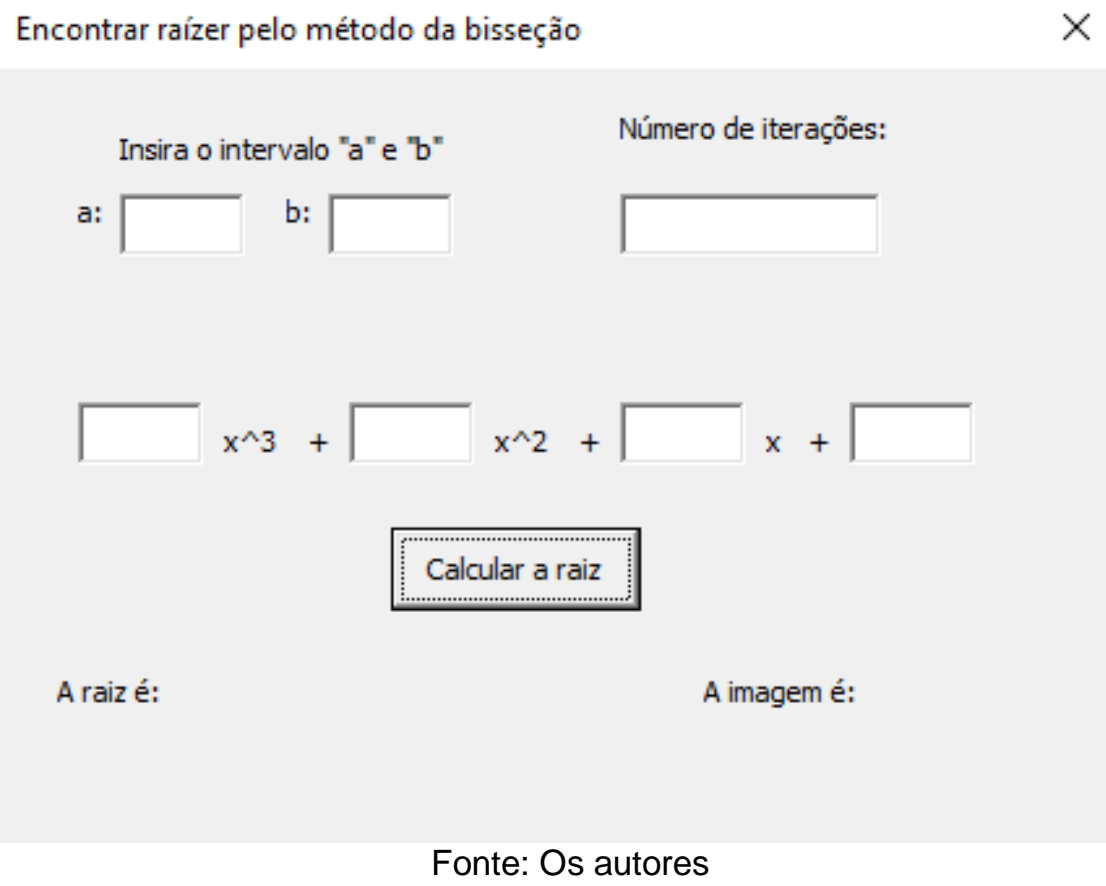

A "Figura 7" ilustra os resultados do questionário a respeito da opinião dos estudantes sobre a usabilidade do programa.

Figura 7 - Pergunta sobre o entendimento do programa. 
Você conseguiu utilizar o aplicativo de maneira adequada ou seja, entendeu o que cada parte significava conforme o avanço da matéria durante o semestre?

3 respostas

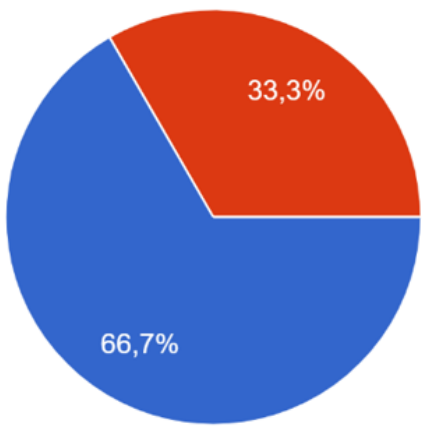

Sim, no começo não sabia muito bem, porém quando o(a) professor(a) passava o conteúdo isso se tornava muito mais fácil.

Sim, porém tiveram métodos que eu não consegui entender o funcionamento no aplicativo mesmo após o(a) professor(a) passar o conteúdo.

Não, compreendi apenas o funcionamento de alguns poucos métodos no programa.

- Não, não consegui relacionar o programa com o que o(a) professor(a) estava ensinando, dessa forma não entendi o funcionamento de nada no programa.

Fonte: Os autores

A segunda pergunta estava relacionada ao interesse no aprendizado e melhora no desempenho na disciplina após os estudantes serem apresentados ao programa, e 66,7\% responderam que acabaram se dedicando mais a disciplina pelo fato de terem recebido acesso ao aplicativo e 33,3\% responderam que provavelmente tiveram seus desempenhos elevados pois estudaram para entender o funcionamento do programa, de acordo com a "Figura 8" que ilustra os resultados da pergunta relacionada a melhora na eficácia na disciplina.

Figura 8 - Pergunta sobre o interesse na disciplina.

Você acha que o fato de você ter sido apresentado ao programa despertou o seu interesse na matéria e ajudou você a ter um desempenho melhor na disciplina de cálculo numérico?

3 respostas

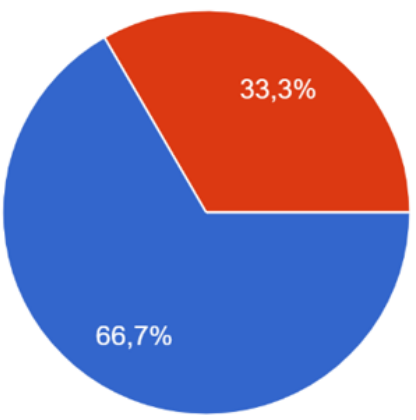

Sim, o programa me despertou o interesse de entender os métodos da matéria, e consequentemente eu acabei estudando mais do que o normal.

Sim, provavelmente o meu desempenho foi melhor do que o esperado, pois eu tive interesse de estudar com 0 intuito de entender o funcionamento do programa.

- Não, provavelmente o aplicativo não me ajudou a ter um desempenho melhor nessa disciplina.

Não, o meu desempenho na disciplina foi menor do que o esperado, e o programa não me despertou $o$ interesse de aprender a matéria.

Fonte: Os autores

No terceiro item do questionário um dos estudantes sugeriu aumentar a quantidade de variáveis nos métodos do programa, algo que é possível de se realizar na programação futuramente. 


\title{
3 CONSIDERAÇÕES FINAIS
}

Diante dos testes, o aplicativo se mostrou fortemente eficaz no processamento e preciso nos resultados, dessa forma foi possível notar o poder da ferramenta até para testes que incluíam manipulações com números muito grandes e também com números muito pequenos, sem demostrar atraso devido a programação pouco eficiente.

Mesmo com as limitações, o aplicativo ainda pode ser de grande ajuda no aprendizado do estudante para o entendimento da disciplina, pois o mesmo pode fazer alterações nos valores de um exercício e rapidamente visualizar no que essas alterações impactam no resultado final.

Apesar dos resultados do questionário ainda se faz necessário realizar testes com um universo maior de estudantes para comprovar a eficácia na melhora do desempenho dos alunos dessa disciplina.

\section{REFERÊNCIAS}

RUGGIERO, Márcia Aparecida Gomes; LOPES, Vera Lúcia da Rocha. Cálculo Numérico. Aspectos teóricos e computacionais. 2ª . edição. São Paulo: Editora Pearson. 2000.

Introdução ao VBA no Office. Disponível em: https://docs.microsoft.com/pt$\mathrm{br} /$ office/vba/library-reference/concepts/getting-started-with-vba-in-office. Acesso em 22 de abr. de 2020.

\section{VISUAL BASIC FOR APPLICATIONS NUMERICAL CALCULATION PROGRAM}

\begin{abstract}
Numerical calculation is the study of methods that serve to obtain the solution of mathematical problems in an approximate way. They invariably involve a large number of arithmetic calculations, making large-scale manual realization impossible. However, with the development of efficient digital computers, it became possible to perform these more extensive calculations quickly and efficiently. Aiming to contribute in a practical and dynamic way in the teaching-learning process of the Numerical Calculus discipline, an application in Visual Basic for Applications (VBA) was developed to solve several typical problems presented in it.
\end{abstract}

Keywords: Algorithm, Application, Programming. 